\title{
Antimicrobial chitosan-PVA hydrogel as a nanoreactor and immobilizing matrix for silver nanoparticles
}

\author{
Shekhar Agnihotri · Soumyo Mukherji • \\ Suparna Mukherji
}

Received: 30 November 2011 / Accepted: 6 March 2012/Published online: 21 March 2012

(c) The Author(s) 2012. This article is published with open access at Springerlink.com

\begin{abstract}
Hydrogels are water-insoluble crosslinked hydrophilic networks capable of retaining a large amount of water. The present work aimed to develop a novel chitosan-PVA-based hydrogel which could behave both as a nanoreactor and an immobilizing matrix for silver nanoparticles (AgNPs) with promising antibacterial applications. The hydrogel containing AgNPs were prepared by repeated freeze-thaw treatment using varying amounts of the crosslinker, followed by in situ reduction with sodium borohydride as a reducing agent. Characterization studies established that the hydrogel provides a controlled and uniform distribution of nanoparticles within the polymeric network without addition of any further stabilizer. The average particle size was found to be $13 \mathrm{~nm}$ with size distribution from 8 to $21 \mathrm{~nm}$ as per HR-TEM studies.
\end{abstract}

S. Agnihotri · Soumyo Mukherji · Suparna Mukherji ( $\square)$ Centre for Research in Nanotechnology and Science (CRNTS), Indian Institute of Technology-Bombay, Powai,

Mumbai 400076, India

e-mail: mitras@iitb.ac.in

S. Agnihotri

e-mail: agnish@iitb.ac.in

\section{Soumyo Mukherji}

Department of Biosciences and Bioengineering,

WRCBB, Indian Institute of Technology-Bombay,

Powai, Mumbai 400076, India

e-mail: mukherji@iitb.ac.in

Soumyo Mukherji

Centre of Excellence in Nanoelectronics,

Indian Institute of Technology-Bombay,

Powai, Mumbai 400076, India

Suparna Mukherji

Centre for Environmental Science and Engineering (CESE),

Indian Institute of Technology-Bombay (IIT-Bombay),

Powai, Mumbai 400076, India
Swelling studies confirmed that higher amount of crosslinker and silver incorporation inside the gel matrices significantly enhanced the porosity and chain entanglement of the polymeric species of the hydrogel, respectively. The AgNP-hydrogel exhibited good antibacterial activity and was found to cause significant reduction in microbial growth (Escherichia coli) in $12 \mathrm{~h}$ while such activity was not observed for the hydrogel without AgNPs.

Keywords Chitosan-PVA hydrogel - Silver nanoparticles · Antibacterial · Swelling study

\section{Introduction}

Hydrogel is a water-insoluble gel-like polymeric network which swells in water due to extensive crosslinking. Its potential to retain significant amount of water is attributed to the presence of several functional groups (i.e., amino, carboxyl, amide, hydroxyl and sulphonic) in the polymers of the hydrogel forming network (Peppas and Khare 1993). Hydrogels usually have well-defined structures that can be modified to yield tailorable functionality and release profile (Mukherji et al. 2012). This has attracted many researchers toward its usage for antibacterial applications either by direct use of the polymer for its antibacterial property or, by incorporating antibacterial agents on the polymeric hydrogel network (Rabea et al. 2003). Chitosan, a natural biopolymer produced in bulk quantity from the shells of crustaceans as byproduct, can be chosen as a promising candidate for making antibacterial hydrogels owing to its innate antibacterial property (Shi et al. 2006). Other favorable properties such as gel forming capability, solubility in dilute acids, and low toxicity make it a suitable material for biomedical purposes. However, the main 
disadvantage associated with chitosan hydrogel is its poor mechanical strength during swelling which may limit its usage for water disinfection purposes. This can be minimized either by physical blending and/or chemical modification by grafting, interpenetrating polymer networks and crosslinking methods (Chandy and Sharma 1992). Chemical crosslinking of chitosan with a polymer having greater mechanical strength, good chemical stability, and hydrophilicity such as polyvinyl alcohol (PVA) may lead to fabrication of a hydrogel composite for antibacterial applications (Zhaoa et al. 2003; Xiao et al. 2010; Hang et al. 2010).

In recent years, there has been growing interest on the usage of silver nanoparticles (AgNPs) for numerous antimicrobial applications, such as, in surgical devices, wound dressings, water disinfection, and antibacterial coatings (Ruparelia et al. 2008; Chen and Schluesener 2008). It is now established that silver nanoparticles exhibit superior antibacterial property than its bulk form and is highly effective against bacteria, virus, and fungi (Li et al. 2010). Most microorganisms find it hard to develop resistance against AgNPs (Galdiero et al. 2011). However, there are some limitations associated with their use for disinfection purposes, when present in aqueous phase. For example, it is difficult to control the shape, size, as well as stability of silver nanoparticles in solution since they undergo rapid oxidation and tend to form aggregates. This may result in reduction of its antimicrobial efficacy at a particular concentration. Also, the cost associated with one time usage of silver nanoparticles and their potential environmental risk to aquatic ecosystem should be taken into consideration. In order to minimize the risk, one promising approach entails the incorporation of silver nanoparticles inside a polymeric matrix that ensures sufficient stabilization with minimum leaching of silver nanoparticles from it.

Till date, several polymeric materials for incorporation of silver nanoparticles have been investigated in the form of microparticles, multilayered films, nanofibers, and hydrogel structures (Zhang et al. 2004; Wu et al. 2009; Yuana et al. 2010; Ma et al. 2009). Although these hybrid materials provide good stability to the synthesized nanoparticles, sincere research efforts have been made to synthesize new hydrogel nanoparticulate system where both the hydrogel and nanoparticle could retain their individual features and characteristics in the combined system (Hamidi et al. 2008; Mohan et al. 2007). In their swollen stage, hydrogels offer large free space within the crosslinked network that can act as a nanoreactor and thus facilitate synthesis of nanoparticles by providing sufficient space for nucleation and growth of nanoparticles (Wang et al. 2004; Mohan et al. 2006, 2007). Inside the hydrogel matrix, silver ions make favorable interactions with the functional moieties present in the polymeric chain and thus, form a uniform distribution inside the whole network (Murthy et al. 2008; Basit et al. 2008). Upon reduction, uniformly distributed silver nanoparticles of controlled size may be achieved which would subsequently immobilize within the polymeric matrix. Recently, a few studies have shown the use of chitosan-PVA hydrogels for antimicrobial and food packaging applications (Tripathi et al. 2009; Hang et al. 2010; Vimala et al. 2011). However, no study has reported their dual role as template-mediated in situ formation and immobilization of silver nanoparticles into the hybrid polymer, for potable water disinfection purposes. Therefore, the aim of the current work is to synthesize and characterize the antimicrobial chitosan-PVA hydrogel which would serve both as a template and an immobilizing matrix for silver nanoparticles. Consequently, the combination of silver nanoparticles within chitosan-PVA polymeric architecture seems to be a promising candidate for the design of new antimicrobial materials.

\section{Materials and methods}

Chitosan $(<90 \%$ deacetylation degree, MW 100,000300,000) was purchased from Marine Chemicals, Kochin, and was used as received. PVA (MW 85,000-124,000) was procured from SD Fine Ltd, Mumbai, and was used without purification. Silver nitrate was obtained from Merck India Pvt Ltd. All other chemicals, i.e., sodium hydroxide, glutaraldehyde solution (25 wt\%), glacial acetic acid, and sodium borohydride were obtained from SD Fine Ltd and were of analytical grade. For antibacterial experiments, the E. coli MTCC443 strain (ATCC 25922) was procured from Institute of Microbial Technology (IMTECH-Chandigarh, India). Deionized water was used throughout the experiments and as polymerization medium during hydrogel formation.

\section{Preparation of chitosan/PVA blended hydrogel}

Chitosan polymer $(2 \% \mathrm{w} / \mathrm{v})$ was dissolved in dilute acetic acid solution at room temperature while stirring overnight. The solution was filtered to remove any impurities and/or undissolved polymer before use. PVA solution (4\% w/v) was prepared by dissolving PVA in deionized water at $80{ }^{\circ} \mathrm{C}$ with stirring for $4 \mathrm{~h}$. Synthesis of chitosan-PVA hydrogel was done in the following manner. A mixture of chitosan and PVA solution was stirred at $60{ }^{\circ} \mathrm{C}$ for $2 \mathrm{~h}$ to obtain a homogenous phase and left overnight with continuous stirring. To achieve network formation, glutaraldehyde was employed as a crosslinker and added to the resulting polymeric mixture. For this, the required amount of glutaraldehyde ( 25 and $50 \mathrm{mM}, 1 \mathrm{ml}$ ) was added slowly to the chitosan-PVA solution at $25{ }^{\circ} \mathrm{C}$ and was mixed well 
for 5 min before casting the resultant solution in the petri dish. The hydrogel membranes were removed from the plate by precipitating in $12 \mathrm{wt} \% \mathrm{NaOH}(\mathrm{aq})$ at room temperature for $1 \mathrm{~h}$, washed thrice with distilled water, then soaked in distilled water for $24 \mathrm{~h}$ and lyophilized. Finally, porous semi-interpenetrating networks were developed by repeated freezing and thawing cycles at $-20{ }^{\circ} \mathrm{C}$ for $6 \mathrm{~h}$ and $25^{\circ} \mathrm{C}$ for $2 \mathrm{~h}$, respectively, for the next 7 days. In this way, two types of chitosan (2\%)/PVA (4\%) hydrogel were prepared with different crosslinking densities achieved using 25 and $50 \mathrm{mM}$ glutaraldehyde.

In situ synthesis and immobilization of silver nanoparticles inside the hydrogel network

Preparation of Ag containing chitosan-PVA hydrogel was performed as follows. Here, in situ reduction of silver ions inside the hydrogel network was done using sodium borohydride as reducing agent. Briefly, various circular discs $(10 \mathrm{~mm}$ diameter) were cut from the prepared hydrogel membrane and were introduced in the $10 \mathrm{mM}$ solution of silver nitrate (silver ion precursor) for $24 \mathrm{~h}$. The silver ion-loaded discs were rinsed twice with deionized water in order to remove the unbound silver ions and were immersed in sodium borohydride solution $(10 \mathrm{mM})$ for $30 \mathrm{~min}$. After complete reduction, the discs were again rinsed using deionized water, lyophilized and kept for further characterization. The schematic representation of silver nanoparticle formation inside the hydrogel network is shown as below.

Swelling studies on the chitosan/PVA blended hydrogel membranes

The chitosan/PVA blended hydrogel membrane was cut into $10-\mathrm{mm}$ circular discs and dried under vacuum for $72 \mathrm{~h}$ to determine their dry weight $\left(W_{2}\right)$. Water content was measured by immersing the chitosan/PVA blended hydrogel membrane in distilled water at $25{ }^{\circ} \mathrm{C}$ for $2 \mathrm{~h}$. The wet weight $\left(W_{1}\right)$ was determined by wiping off the surface water with a piece of filter paper. The absorbed water content was then calculated by determining the water content in term of the swelling ratio $(S \%)$ using Eq. 1:

$S(\%)=\frac{W_{1}-W_{2}}{W_{1}} \times 100$.

Characterization of hydrogel composites

The Fourier transform infrared (FTIR) spectra of pure chitosan-PVA and Ag immobilized Chitosan-PVA were recorded with an FTIR-Spectrophotometer (MAGNA 550, Nicolet Instruments Corporation, USA) using $\mathrm{KBr}$ pellet. UV-visible spectra of the silver nanoparticles from the nanocomposite hydrogel were recorded using a spectrophotometer (Perkin-Elmer, CA) in the absorbance mode (range 200-800 nm). The morphology of hydrogel membrane was observed by FEG-SEM (JEOL JSM-7600, Japan) with platinum coating using a sputter system. The particle size and size distribution of AgNPs in the composite hydrogel was determined using HR-TEM (JEOL JEM 2100F, Japan) at an operational voltage of $200 \mathrm{kV}$. Mass loading of AgNP inside the hydrogel was determined using inductively coupled plasma-atomic emission spectrometer (ARCOS ICP-AES spectro, Germany) using the following procedure. Initially, a pre-weighed hydrogel was crushed into powdered form and was immersed in $5 \mathrm{ml}$ of deionized water overnight. The solution was then sonicated for $2 \mathrm{~h}$, treated with nitric acid $(70 \%, 200 \mu \mathrm{l})$ to ensure dissolution of the AgNPs and finally filtered (Whatman 40) to remove the hydrogel debris. Various dilutions were tested using silver standards in the range of $10 \mathrm{ppb}$ to $100 \mathrm{ppm}$. The presence of residual boron in the hydrogel was also investigated through ICP-AES. The study was conducted in duplicate.

Antibacterial activity of the hydrogel

The antibacterial activity of these hydrogel nanocomposites was determined using E. coli MTCC443 strain in nutrient broth medium. The sterilized medium was inoculated using $10 \mu \mathrm{l}$ of microbial culture and kept in the rotary shaker at $150 \mathrm{rpm}, 37^{\circ} \mathrm{C}$ for $16 \mathrm{~h}$. At the mid-log phase of microbial growth, cells were centrifuged, washed thrice and resuspended in $2 \%$ phosphate buffer. The final cell concentration was adjusted to unit absorbance at $600 \mathrm{~nm}$ using UV spectrophotometer which corresponded to $1-2 \times 10^{9} \mathrm{CFU} / \mathrm{ml}$. The antibacterial activity of the composite hydrogel was tested by immersing the hydrogel discs (10 $\mathrm{mm}$ diameter, 2 in number) into the microbial solution and incubating under aforementioned conditions. Samples were withdrawn from the test solution at different time intervals for determining the biocidal action of the composite hydrogel. Pure chitosan-PVA hydrogel was also used as a control for the antibacterial study. The growth kinetics of pure culture solution (devoid of hydrogel and silver nanoparticles) was also employed as a negative control.

\section{Results and discussion}

Template-mediated in situ formation of silver nanoparticles in the hydrogel

Due to the importance of chitosan-silver nanofilms/membranes in antimicrobial application, water purification and 
wound dressing applications, a chitosan/PVA hydrogel was developed to act both as a nanoreactor as well as a template for immobilization of silver nanoparticles. The hydrogel networks acted as a carrier in the reaction process and subsequently behaved as an immobilizing matrix for the synthesized nanoparticles (Mohan et al. 2007). An uniform distribution of silver nanoparticles inside the hydrogel could be achieved due to its tendency to form complex and/ or non-covalent interactions with silver ions and the functional moieties present in the hydrogel network such as $-\mathrm{OH},-\mathrm{NH}_{2},-\mathrm{C}=\mathrm{O}$ (Tarnavchyk et al. 2009; Bozanic et al. 2010; Mohan et al. 2010). The addition of the fully swollen hydrogel to silver nitrate solution caused adsorption/ anchoring of $\mathrm{Ag}^{+}$ions with the functional groups via ionexchange mechanism. Also, many silver ions may get trapped in the free spaces between the crosslinked polymeric networks (Mohan et al. 2010). Thus, reduction of silver ions using any reducing agent results in the formation of silver nanoparticles within as well as in the free spaces between the polymeric chains. In the present approach, silver-loaded hydrogel networks were reduced in situ with $\mathrm{NaBH}_{4}$ to form highly dispersed silver nanoparticles. Since sodium borohydride is a strong reducing agent, the color of the hydrogel changed to deep yellow within $10 \mathrm{~s}$ which indicated the formation of silver nuclei. However, color of the discs changed gradually from yellow to light brown and finally to deep brown over time (30 $\mathrm{min}$ ) which indicated the formation of silver nanoparticles, as shown in Fig. 1.

It is reported that, change in concentration of the crosslinking agent may affect the free space between the polymeric networks, and may affect the size, shape or distribution of the silver nanoparticles within the hydrogel. This can be achieved either by altering the crosslinker amount or by choosing different types of crosslinker, often in different combinations. A highly dense hydrogel network is desired which favors the intra-inter molecular interactions between the silver ions and the gel network, resulting in the formation of smaller size silver nanoparticles. In contrast, a hydrogel with high porosity provides large surface area for silver nuclei generation and thus, produces a large number of silver nanoparticles with its potential to limit their aggregation. The porosity affords an opportunity for the rapid diffusion of water molecules into and out of the polymers and this property may be important for antibacterial applications. Therefore, the crosslinker density appears to be a key factor for in situ formation and immobilization of silver nanoparticles inside the hydrogel.

Considering the toxic effects of glutaraldehyde, a minimum amount of crosslinker ( 25 and $50 \mathrm{mM}$ ) was used in forming the hydrogels. In a study by Spencer et al. (1978), male rats when exposed to $0.25 \%$ glutaraldehyde (i.e., $25 \mathrm{mM}$ ) in their drinking water for 11 weeks exhibited no evidence of toxicity or damage to the peripheral or central nervous systems. Several studies have reported effective removal of unused glutaraldehyde by repeated washing with deionized water (Hoffmann et al. 2009). Moreover, in some established protocols for microscopic fixation, unreacted glutaraldehyde is completely removed by treatment with sodium borohydride (Gard and Kropf 1993). In the current study, the synthesized hydrogels were extensively washed with DI water to remove the unreacted glutaraldehyde. Subsequently, an excess of sodium borohydride was used during in situ AgNP formation. This would further ensure complete removal of glutaraldehyde from the hydrogel. Thus, the chitosan-PVA hydrogels synthesized using two different concentrations of glutaraldehyde, i.e., 25 and $50 \mathrm{mM}$ (designated as CP-25 and CP-50, respectively) are not expected to contain any unreacted glutaraldehyde that may contribute to toxicity.

\section{Swelling studies}

The impact of silver nanoparticle incorporation on the chitosan-PVA hydrogel and crosslinking densities on the swelling behavior was evaluated as shown in Table 1 . The results showed that for 25 and $50 \mathrm{mM}$ crosslinker concentration, pure hydrogel had swelling ratios of 11 and $26 \%$,

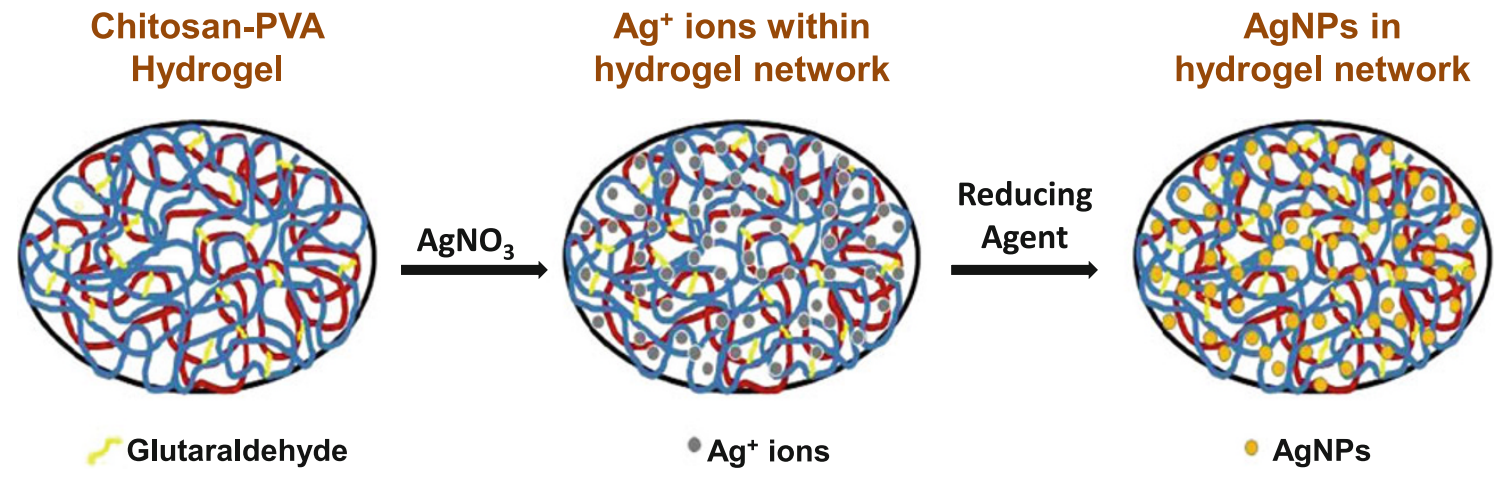

Fig. 1 Schematic representation of in situ synthesis of silver nanoparticles inside the chemically crosslinked hydrogel network 
respectively, while for Ag-loaded hydrogel it decreased to 5 and $22 \%$, respectively. This can be explained on the basis of additional cross-links between AgNPs and the electron-rich $\mathrm{O}$ and $\mathrm{N}$ atoms present in the polymeric chains which restrained the water penetration ability of the hydrogel and decreased the overall swelling behavior (Vimala et al. 2011). In another study, Ma et al. (2009) established that formation of silver nanoparticles inside the polymeric matrix enhanced the flexibility of the hydrogel network due to stronger interactions between $\mathrm{Ag}$ and the hydrophilic moieties present in the polymer. The resulting decrease in hydrophilicity of the composite hydrogel reduced the swelling behavior. Swelling studies also revealed that a higher concentration of the crosslinker could significantly increase the porosity and template area for nanoparticle formation, resulting in an enhanced swelling ratio. Wang and Gunasekaran (2006) studied the effect of crosslinker amount on the swelling ratio of the hydrogel and postulated that higher crosslinker amount leads to higher degree of localization of water molecules inside the hydrogel network resulting in an increase in the swelling ratio. This can be explained on the basis of stronger interactions and flexible chain entanglements between the two polymers blended as the crosslinker amount is increased.

Figure 2 shows the swelling kinetics of chitosan hydrogels in deionized water $(\mathrm{pH} 7)$ at room temperature.

Table 1 Swelling ratios of the synthesized hydrogels with different glutaraldehyde concentration

\begin{tabular}{llc}
\hline Hydrogel & $\begin{array}{l}\text { Swelling ratio } \\
\text { (pure hydrogel) }(\%)\end{array}$ & $\begin{array}{l}\text { Swelling ratio } \\
\text { (hydrogel }+ \text { AgNPs) }(\%)\end{array}$ \\
\hline CP-25 & $11.63 \pm 0.18$ & $5 \pm 0.22$ \\
CP-50 & $25.85 \pm 0.38$ & $22 \pm 0.64$ \\
\hline
\end{tabular}

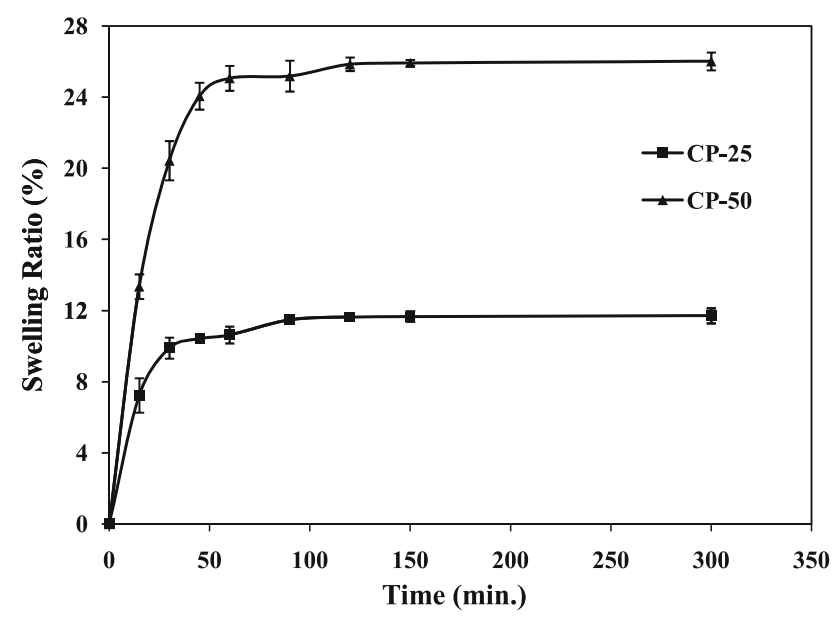

Fig. 2 Swelling kinetics of chitosan-PVA hydrogel at $\mathrm{pH} 7$, room temperature with $25 \mathrm{mM}$ (CP-25) and $50 \mathrm{mM}$ (CP-50) glutaraldehyde as crosslinker. Experiments were performed in duplicate and the error bars represent standard deviation
For both $\mathrm{CP}-25$ and $\mathrm{CP}-50$, there was a gradual increase in the swelling ratio up to $120 \mathrm{~min}$ after which no significant change was observed. Swelling ratio at this stage was termed as the equilibrium swelling ratio, whose value was $11.63 \pm 0.18$ and $25.85 \pm 0.38 \%$ for CP-25 and CP-50 hydrogel, respectively. However, it should be noted that the equilibrium swelling ratio is a temperature-dependent property which varies with formation or breakup of hydrogen bonds between hydroxyl groups of PVA and amino groups present within the chitosan polymeric network (Kim et al. 2003).

On the basis of higher hydrophilic nature, CP-50 hydrogel was chosen over CP-25 for further characterizations and antimicrobial study. Chung et al. (2004) illustrated a correlation between hydrophilic nature of the chitosan and its antibacterial activity against Gram negative bacteria, i.e., E. coli.

\section{Characterization of synthesized chitosan-PVA hydrogel}

\section{FTIR studies}

The FTIR spectrum of synthesized chitosan-PVA hydrogel (CP-50) and nano-silver-loaded hydrogel was evaluated (Fig. 3). Result showed that the synthesized hydrogels displayed good compatibility between chitosan and PVA polymer with significant changes observed after silver nanoparticle incorporation. A peak appeared at $3,445 \mathrm{~cm}^{-1}$ indicating the presence of hydrogen bonds between chitosan and PVA polymers causing $\mathrm{OH} / \mathrm{NH}_{2}$ stretching (Vimala et al. 2011). Other peaks, i.e., 1,721, 1,635 and $1,418 \mathrm{~cm}^{-1}$ may be attributed to carbonyl group stretching, absorption of $\mathrm{NHCOCH}_{3}$ groups and $\mathrm{C}-\mathrm{H}$ bonds present, respectively, in the chitosan/PVA polymeric chains (Lin et al. 2011; Yang et al. 2004; Vimala et al. 2011). The appearance of carbonyl group in chitosan/PVA hydrogel, even after crosslinking with glutaraldehyde indicates free $-\mathrm{OH}$ moieties of PVA due to the incomplete reaction of glutaraldehyde with hydroxyl groups of PVA (Mansur et al. 2008). These hydroxyl groups therefore represent ideal sites for the anchoring of silver ions, contributing later to the silver nanoparticle immobilization. Interestingly, the absorption peaks were observed at 2,955 and $2,860 \mathrm{~cm}^{-1}$ in both pristine and the nano-silver-loaded hydrogels, which originate from $\mathrm{CH}_{2}$ asymmetric stretching and $\mathrm{C}-\mathrm{H}$ bond of chitosan/PVA and chitosan chains, respectively (Xiao et al. 2010; Vimala et al. 2011). However, their intensity was increased in nano-silver-loaded hydrogel, which confirmed that the incorporation of silver nanoparticles could enhance stretching of the polymeric backbone chain, resulting in an increase in the flexibility of the hydrogel, as also demonstrated by the swelling studies. 
Fig. 3 FTIR spectrum of pure chitosan/PVA hydrogel (CP-50) and silver nanoparticle-loaded hydrogel

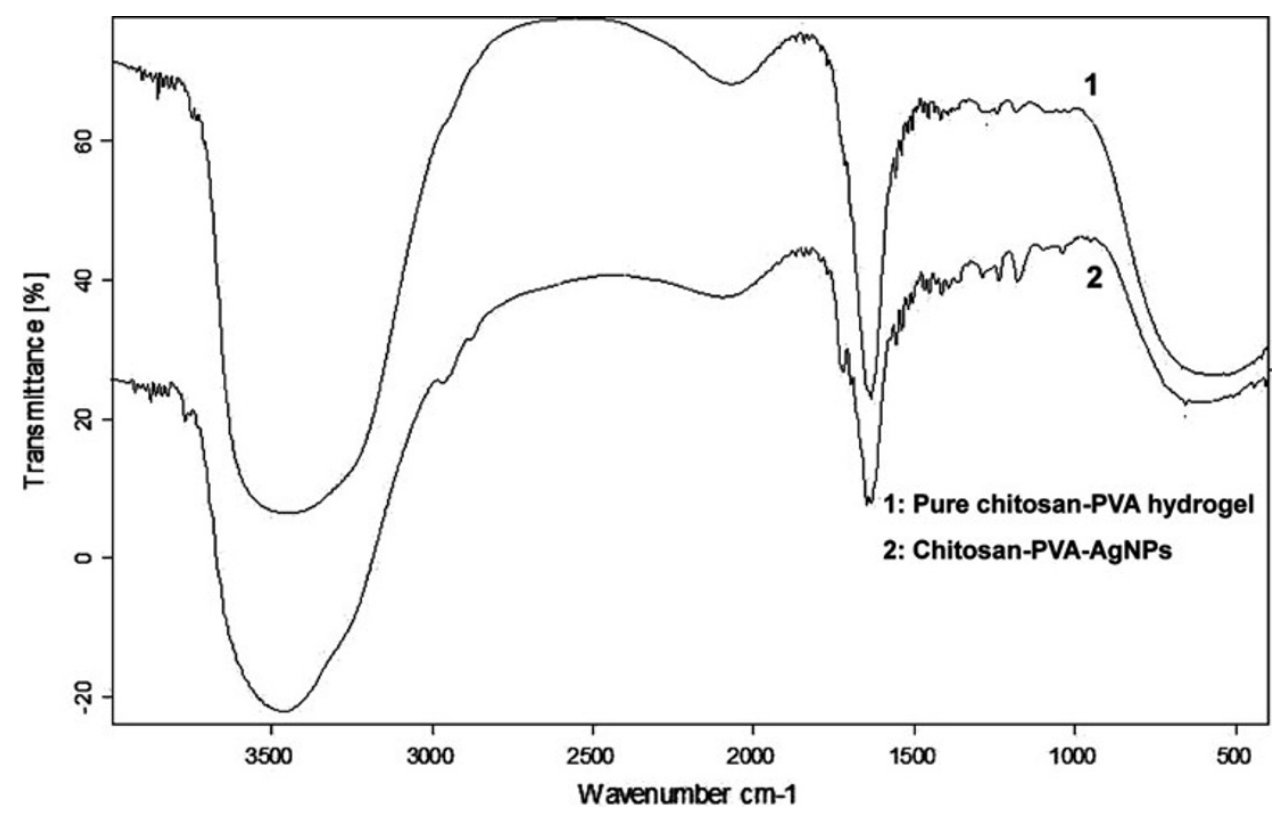

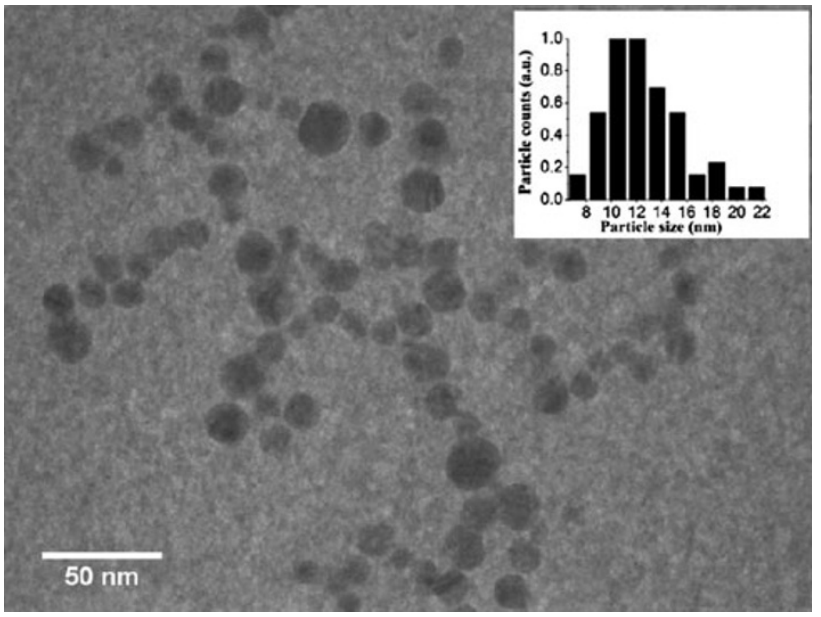

Fig. 5 HR-TEM images of the synthesized silver nanoparticle inside chitosan-PVA hydrogel (CP-50). Inset shows the size distribution of nanoparticles

behavior to differ from those of isolated nanoparticles. To overcome the possibility of hindrance within the immobilizing network, silver nanoparticles were retrieved from the hydrogel structure as described by Thomas et al. (2009). AgNP containing hydrogel films (CP-50/nano-Ag) were sonicated in deionized water so as to facilitate the relaxation of polymeric chain entanglement within the hydrogel network. As a result, mesh size of the swollen gel increased, ensuring recovery of some of the silver nanoparticles in colloidal form. Interestingly, these nanoparticles displayed a sharp peak at $400 \mathrm{~nm}$ which confirmed the presence of small-sized nanoparticles with a narrow size distribution. This blue shift of $28 \mathrm{~nm}$ in the absorption maxima indicated that the actual size of silver nanoparticles would be even smaller than that 
expected through the UV-vis study. Thus, HR-TEM analysis was performed further to identify the exact size and distribution of the silver nanoparticles synthesized inside the hydrogel films. However, in both spectra no absorption peak was observed at 335 and $560 \mathrm{~nm}$ which indicated the absence of any AgNP aggregates and silver clusters within the hydrogel (Gutiérrez and Henglein 1993; Ma et al. 2009).

\section{HR-TEM studies}

HR-TEM study provides valuable information regarding size and distribution of AgNP within hydrogel nanocomposites.
Figure 5 indicated that the average size of AgNP was $13.3 \pm 3.8 \mathrm{~nm}$ with size distribution in the range of 8-21 nm (inset). No aggregates of nanoparticles were observed.

\section{FEG-SEM studies}

The cross-sectional morphology of the hydrogels was observed to evaluate in situ formation of silver nanoparticles inside the hydrogel networks. As shown in Fig. 6a, b, pristine hydrogel (CP-50) did not show any particles in the FEG-SEM image. Loading of silver nanoparticles inside the hydrogel did not affect its morphology and structural
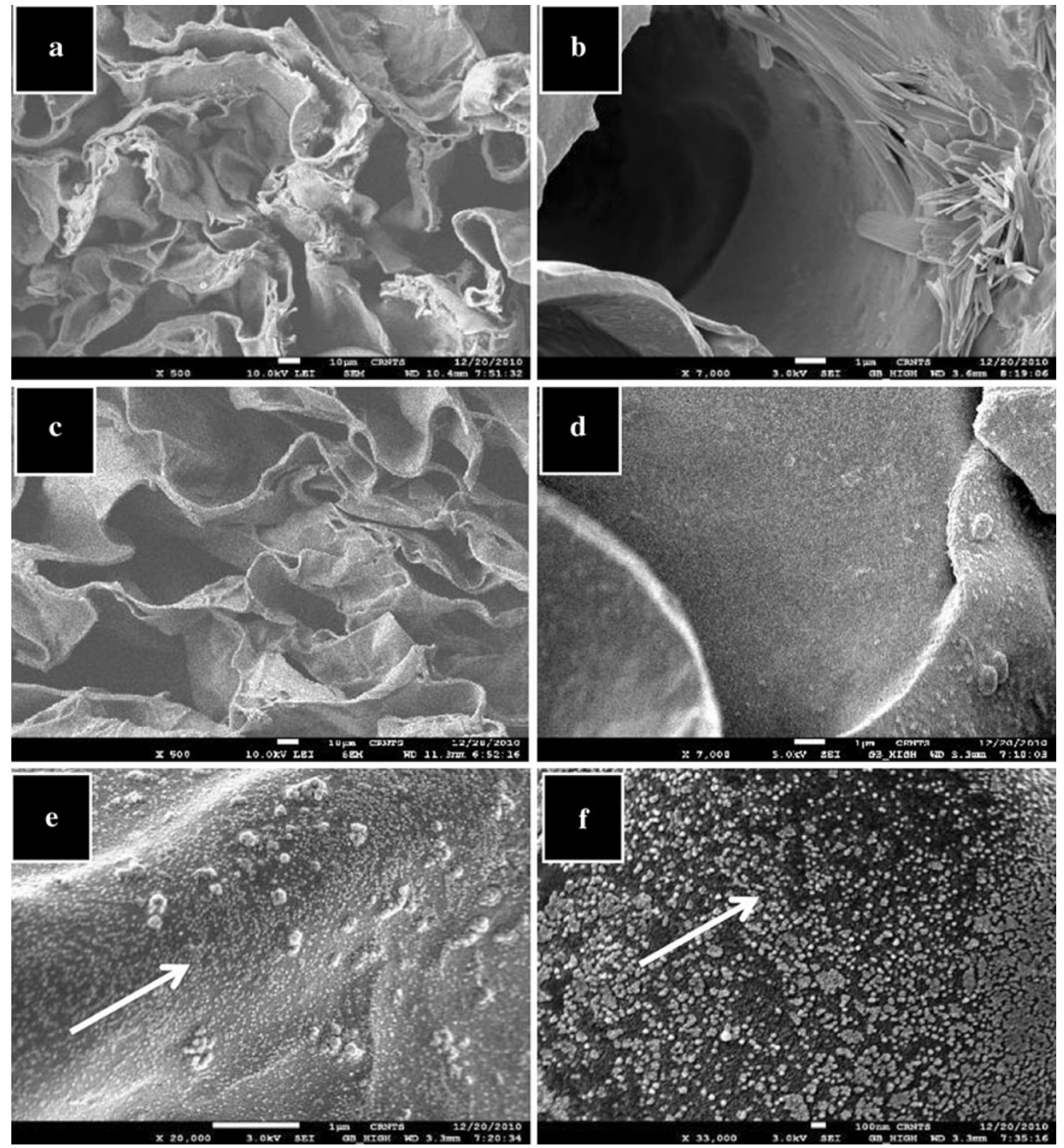

Fig. 6 FEG-SEM images of pure chitosan-PVA hydrogel $(\mathrm{CP}-50)$ at $\mathbf{a} \times 500$ and $\mathbf{b} \times 7,000$ magnification. $\mathbf{c}-\mathbf{f}$ Demonstrated Ag-loaded chitosan-PVA hydrogel at different magnifications: $\mathbf{c} \times 500, \mathbf{d} \times 7,000, \mathbf{e} \times 20,000, \mathbf{f} \times 33,000$

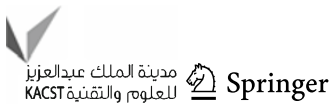


integrity (Fig. 6c, d). Figure 6e, f showed that silver nanoparticles were evenly distributed between the polymeric chain networks with a size range of $25-35 \mathrm{~nm}$. This corroborates with the UV absorption maxima at $428 \mathrm{~nm}$ which corresponds to silver nanoparticles of similar size range as shown by Hutter and Fendler (2004). However, it is not possible to judge the actual size of impregnated silver nanoparticles inside the hydrogels from FEG-SEM results where analysis is performed on dried hydrogel samples as discussed by Mohan et al. (2010). Thus, the size of silver nanoparticles determined using HR-TEM studies, i.e., $13.3 \mathrm{~nm}$ was considered to be more appropriate for this study. As shown by the FEG-SEM results, the main advantage of the current technique is that it provided a controlled and uniform distribution of nanoparticles within the hydrogel network without addition of any other stabilizer. It was expected that a homogeneous distribution of silver nanoparticles would be achieved throughout the hydrogel networks, although there was a rapid reduction of the silver ions into the silver nanoparticles with sodium borohydride. The reason is that the silver nanoparticles initially formed in the network causes expansion of the free space in the hydrogel, since silver ions interact electrostatically with hydroxyl groups of the PVA moieties. This made it possible for $\mathrm{NaBH}_{4}$ to enter into the entire gel network to reduce the silver ions present in the network. Nevertheless, the presence of intra- and inter-molecular forces between chitosan and polyvinyl alcohol units facilitated formation of an extensive physical network of hydrogen bonds, which provided 'nano' domains for growth of the nanoparticles as well as ensured their protection within the network. Furthermore, chitosan chains in the hydrogel possibly participated in the reduction process, as also suggested by previous studies (Vimala et al. 2011).

Amount of silver in the hydrogel

The amount of silver nanoparticles loaded inside the hydrogel was evaluated using ICP-AES. Crushing of the hydrogel and sonication were used to completely retrieve AgNPs from the interior of the hydrogel matrix while nitric acid digestion was used to ensure dissolution of the AgNPs. The solution obtained after extracting and dissolving the AgNPs loaded on a hydrogel $(0.218 \mathrm{~g})$ in $5 \mathrm{ml}$ acidified deionized water contained $22.3 \pm 2.3 \mathrm{ppm}$ of silver and $19.2 \pm 4.7 \mathrm{ppb}$ of boron based on ICP studies. Thus, the CP-50 hydrogel contained $0.51 \pm 0.06 \mathrm{mg}$ of $\mathrm{AgNP} / \mathrm{g}$ of CP-50 hydrogel $(0.05 \% \mathrm{AgNP})$. This confirms significant immobilization of AgNPs within the hydrogel network. Boron constituted less than $0.1 \%$ of the mass of silver nanoparticles immobilized inside the hydrogel. Thus, traces of boron impurity exist and it cannot be completely

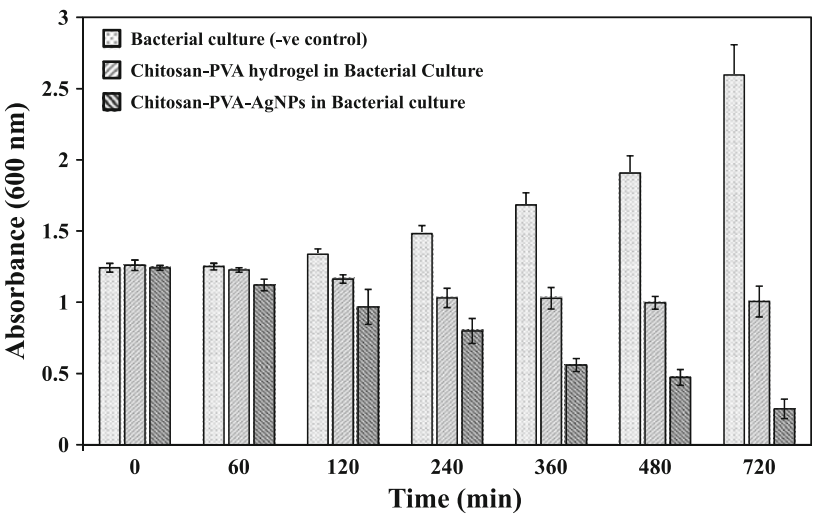

Fig. 7 Antibacterial test showing the antimicrobial efficacy of pure chitosan-PVA hydrogel and AgNP loaded chitosan-PVA hydrogel. Error bars represent standard deviation for studies conducted in duplicate

excluded as also reported in other studies (Ruparelia et al. 2008).

Antibacterial studies

The antibacterial action of synthesized hydrogel (CP-50) is an important factor for determining its application in water disinfection devices. Here, the pure hydrogel and nano-Agloaded hydrogels tested against $E$. coli MTCC443 strain showed a significant reduction in the bacterial growth over an incubation period of $720 \mathrm{~min}$ (Fig. 7). After $12 \mathrm{~h}$, there was $83.5 \%$ reduction in E. coli growth for Ag-incorporated hydrogel compared to $24.4 \%$ reduction for the pure hydrogel. Moreover, it was found that silver-immobilized hydrogels showed superior antimicrobial efficacy compared to its pure form at all sampling times. The presence of the porous hydrogel facilitates frequent diffusion of liquid media across the hydrogel which could release silver nanoparticles into it (Vimala et al. 2011). However, the basis for growth inhibition in pure chitosan-PVA was due to the inherent antimicrobial nature of the chitosan polymer present in the hybrid network (Shi et al. 2006). Polycationic chitosan can interact with the negatively charged moieties present on the bacterial surface causing membrane leakage, loss in cell permeability and cell death. Therefore, porous hydrogel-silver nanocomposites exhibited good antimicrobial activity and displayed enhanced biocidal efficacy for disinfection purposes.

\section{Conclusion}

A porous, chemically crosslinked chitosan-PVA hydrogel was prepared by repeated freeze-thaw treatment for promising antibacterial applications. The hydrogel is an 
ideal candidate for the successful in situ synthesis of silver nanoparticles within the polymeric networks, which also serve as an immobilizing matrix. Morphology studies (FEG-SEM, FEG-TEM) indicate that AgNPs are located on the surface as well as interior of the hydrogel and an effective incorporation of metal nanoparticles into the hydrogel network was achieved. An additional advantage is that a uniform distribution of silver nanoparticles with narrow size distribution was achieved without use of any stabilizer. Swelling studies also revealed that a higher crosslinker concentration and incorporation of silver nanoparticles enhanced the overall morphology of the hydrogel structure, i.e., they imparted higher porosity and flexibility to the polymeric chain units. The developed nanocomposite hydrogel showed effective biocidal action against $E$. coli within $12 \mathrm{~h}$, thus showing great potential for use in water disinfection purposes.

Acknowledgments The authors would like to acknowledge use of research facilities at Centre for Research in NanoTechnology and Sciences (CRNTS), and Sophisticated Analytical Instrumentation Facility (SAIF), IIT-Bombay. The antimicrobial experiments were conducted at the Centre for Environmental Science and Engineering (CESE), IIT-Bombay. Financial support from the DST-funded project [Centre for Nanotechnology (Polymer nanosensors, Nanobiotechnology, and Nanoelectronics)] is greatly acknowledged.

Open Access This article is distributed under the terms of the Creative Commons Attribution License which permits any use, distribution, and reproduction in any medium, provided the original author(s) and the source are credited.

\section{References}

Basit H, Pal A, Sen S, Bhattacharya S (2008) Two-component hydrogels comprising fatty acids and amines: structure, properties, and application as a template for the synthesis of metal nanoparticles. Chem Euro J 14(21):6534-6545

Bozanic DK, Trandafilovic LV, Luyt AS, Djokovic V (2010) Green synthesis and optical properties of silver-chitosan complexes and nanocomposites. React Funct Polym 70:869-873

Chandy T, Sharma CP (1992) Prostaglandin $E_{1}$-immobilized poly (vinyl alcohol)-blended chitosan membranes: Blood compatibility and permeability properties. J Appl Polym Sci 44(12):21452156

Chen X, Schluesener HJ (2008) Nanosilver: a nanoproduct in medical application. Toxicol Lett 176:1-12

Chung YC, Su YP, Chen CC, Ji G, Wang HL, Wu Gaston JC, Lin JG (2004) Relationship between antibacterial activity of chitosan and surface characteristics of cell wall. Acta Pharmacol Sin 25(7):932-936

Galdiero S, Falanga A, Vitiello M, Cantisani M, Marra V, Galdiero M (2011) Silver nanoparticles as potential antiviral agents. Molecules 16:8894-8918

Gard DL, Kropf DL (1993) Confocal immunofluorescence microscopy of microtubules in oocytes, eggs, and embryos, of algae and amphibians. Methods Cell Biol 37:147-169

Gutiérrez M, Henglein A (1993) Formation of colloidal silver by "Push-Pull" reduction of $\mathrm{Ag}^{+}$. J Phys Chem B 97:11368-11370
Hamidi M, Azadi A, Rafiei P (2008) Hydrogel nanoparticles in drug delivery. Adv Drug Del Rev 60:1638-1649

Hang AT, Tae B, Park JS (2010) Non-woven mats of poly(vinyl alcohol)/chitosan blends containing silver nanoparticles-fabrication and characterization. Carbohydr Polym 82:472-479

Hoffmann B, Seitz D, Mencke A, Kokott A, Ziegler G (2009) Glutaraldehyde and oxidised dextran as crosslinker reagents for chitosan-based scaffolds for cartilage tissue engineering. J Mater Sci Mater Med 20(7):1495-1503

Hutter E, Fendler JH (2004) Exploitation of localized surface plasmon resonance. Adv Mater 16(19):1685-1706

Kim SJ, Park SJ, Kim SI (2003) Swelling behavior of interpenetrating polymer network hydrogels composed of poly(vinyl alcohol) and chitosan. React Funct Polym 55(1):53-59

Li WR, Xie XB, Shi QS, Zeng HY, Ou-Yang YS, Chen YB (2010) Antibacterial activity and mechanism of silver nanoparticles on Escherichia coli. Appl Microbiol Biotechnol 85(4):1115-1122

Lin SB, Yuan CH, Ke AR, Li YL, Ouyang N (2011) Electrical sensitivity and mechanical properties of fast responsive PAMPS-PAA-PVA T-IPN hydrogels. Adv Poly Technol. doi: 10.1002/adv.20266

Ma YQ, Yi JZ, Zhang LM (2009) A facile approach to incorporate silver nanoparticles into dextran-based hydrogels for antibacterial and catalytical application. J Macromol Sci Part A Pure Appl Chem 46(6):643-648

Mansur H, Sadahira C, Souza A, Mansur A (2008) FTIR spectroscopy characterization of poly(vinyl alcohol) hydrogel with different hydrolysis degree and chemically crosslinked with glutaraldehyde. Mater Sci Eng C 28(4):539-548

Mohan MY, Premkumar T, Lee K, Geckeler KE (2006) Fabrication of silver nanoparticles in hydrogel networks. Macromol Rapid Commun 27:1346-1354

Mohan MY, Lee K, Premkumar T, Geckeler KE (2007) Hydrogel networks as nanoreactors-a novel approach to silver nanoparticles for antibacterial applications. Polymer 48:158-164

Mohan YM, Vimala K, Thomas V, Varaprasad K, Sreedhar B, Bajpai SK, Raju KM (2010) Controlling of silver nanoparticles structure by hydrogel networks. J Colloid Interface Sci 342:73-82

Mukherji S, Ruparelia J, Agnihotri S (2012) Antimicrobial activity of silver and copper nanoparticles: variation in sensitivity across various strains of bacteria and fungi. In: Cioffi N, Rai M (eds) Nano-antimicrobials-progress and prospects, part-2, Springer, pp 225-251. doi:10.1007/978-3-642-24428-5-8

Murthy PSK, Mohan YM, Varaprasad K, Sreedhar B, Raju KM (2008) First successful design of semi-IPN hydrogel-silver nanocomposites: a facile approach for antibacterial application. J Colloid Interface Sci 318(2):217-224

Peppas NA, Khare AR (1993) Preparation, structure and diffusional behavior of hydrogels in controlled release. Adv Drug Del Rev 11(1-2):1-35

Rabea EI, Badawy MET, Stevens CV, Smagghe G, Steurbaut W (2003) Chitosan as antimicrobial agent: applications and mode of action. Biomacromolecules 4:1457-1465

Ruparelia JP, Duttagupta SP, Chatterjee AK, Mukherji S (2008) Strain specificity in antimicrobial activity of silver and copper nanoparticle. Acta Biomater 4(3):707-716

Shi ZL, Neoha KG, Kanga ET, Wang W (2006) Antibacterial and mechanical properties of bone cement impregnated with chitosan nanoparticles. Biomaterials 27:2440-2449

Spencer PS, Bischoff MC, Schaumburg HH (1978) On the specific molecular configuration of neurotoxic aliphatic hexacarbon compounds causing central-peripheral distal axonopathy. Toxicol Appl Pharmacol 44:17-28

Tarnavchyk I, Voronov A, Kohut A, Nosova N, Varvarenko S, Samaryk V, Voronov S (2009) Reactive hydrogel networks for

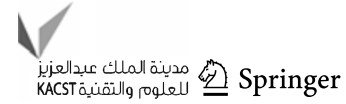


the fabrication of metal-polymer nanocomposites. Macromol Rapid Commun 30(18):1564-1569

Thomas V, Yallapu MM, Sreedhar B, Bajpai SK (2009) Breathing-in/ breathing-out approach to preparing nanosilver-loaded hydrogels: highly efficient antibacterial nanocomposites. J Appl Polym Sci 111:934-944

Tripathi S, Mehrotra GK, Dutta PK (2009) Physicochemical and bioactivity of crosslinked chitosan-PVA film for food packaging applications. Int J Biol Macromol 45(4):372-376

Vimala K, Mohan YM, Varaprasad K, Redd NN, Ravindra S, Naidu NS, Raju KM (2011) Fabrication of curcumin encapsulated Chitosan-PVA silver nanocomposite films for improved antimicrobial activity. J Biomater Nanobiotechnol 2:55-64

Wang T, Gunasekaran S (2006) State of water in chitosan-PVA hydrogel. J Appl Polym Sci 101:3227-3232

Wang C, Flynn NT, Langer R (2004) Controlled structure and properties of thermoresponsive nanoparticle-hydrogel composites. Adv Mater 16(13):1074-1079
Wu Y, Jia W, An Q, Liu Y, Chen J, Li G (2009) Multiaction antibacterial nanofibrous membranes fabricated by electrospinning: an excellent system for antibacterial applications. Nanotechnology 20(24):245101

Xiao C, Gao F, Gao Y (2010) Controlled preparation of physically crosslinked chitosan-g-poly(vinyl alcohol) hydrogel. J Appl Polym Sci 117:2946-2950

Yang JM, Su WY, Leu TL, Yang MC (2004) Evaluation of chitosan/ PVA blended hydrogel membranes. J Memb Sci 236:39-51

Yuana W, Fua J, Sua K, Ji J (2010) Self-assembled chitosan/heparin multilayer film as a novel template for in situ synthesis of silver nanoparticles. Coll Surf B Biointerface 76:549-555

Zhang J, Xu S, Kumacheva E (2004) Polymer microgels: reactors for semiconductor, metal, and magnetic nanoparticles. J Am Chem Soc 126:7908-7914

Zhaoa L, Mitomoa H, Zhaib M, Yoshiic F, Nagasawac N, Kume T (2003) Synthesis of antibacterial PVA/CM-chitosan blend hydrogels with electron beam irradiation. Carbohydr Polym 53:439-446 